\title{
Numerical Stability of Explicit Off-lattice Boltzmann Schemes: A comparative study
}

\author{
Parthib R. Rao ${ }^{1}$, Laura A. Schaefer \\ Department of Mechanical Engineering and Material Science, University of Pittsburgh, \\ 636 Benedum Hall, 3700 O'Hara St., \\ Pittsburgh PA, 15261, USA.
}

\begin{abstract}
The off-lattice Boltzmann (OLB) method consists of numerical schemes which are used to solve the discrete Boltzmann equation. Unlike the commonly used lattice Boltzmann method, the spatial and time steps are uncoupled in the OLB method. In the currently proposed schemes, which can be broadly classified into Runge-Kutta-based and characteristics-based, the size of the time-step is limited due to numerical stability constraints. In this work, we systematically compare the numerical stability of the proposed schemes in terms of the maximum stable time-step. In line with the overall LB method, we investigate the available schemes where the advection approximation is explicit, and the collision approximation is either explicit or implicit. The comparison is done by implementing these schemes on benchmark incompressible flow problems such as Taylor vortex flow, Poiseuille flow and, lid-driven cavity flow. It is found that the characteristics-based OLB schemes are numerically more stable than the Runge-Kutta-based schemes. Additionally, we have observed that, with respect to time-step size, the scheme proposed by Bardow et al. [? ] is the most numerically stable and computationally efficient scheme compared to similar schemes, for the flow problems tested here.
\end{abstract}

Keywords: Off-lattice Boltzmann method, finite-difference, numerical stability

\section{Introduction}

The lattice Boltzmann (LB) method is an alternative and powerful numerical technique used for modeling a variety of complex hydrodynamic flows [? ? ]. Unlike conventional numerical methods which discretize the macroscale governing equations directly, the LB method solves a fully-discrete kinetic equation for distribution functions (DFs) $f_{i}(\boldsymbol{x}, t)$, designed to reproduce the Navier-Stokes equation in the hydrodynamic limit. The LB method has advantages such as ease of parallelization, simplicity of programming, and a capability for incorporating model interactions for simulating complex flows.

A defining feature of the LB method is the coupling between the velocity and space-time discretizations. That is, for a particular discrete-velocity set, $\boldsymbol{\xi}_{i}$, the coupling automatically fixes the temporal and spatial steps through the relation $\Delta \boldsymbol{x}=\boldsymbol{\xi}_{i} \Delta t$. This procedure has some advantages such as numerical-diffusion free (exact) advection and computational efficiency (copy-operation). The coupling is, in fact, a carryover from the earliest LB models, which were based on Lattice Gas Automata (LGA). However, the LGA link was broken when it was shown more than a decade ago that the LB method can be derived directly from the

Email addresses: prr28@pitt.edu (Parthib R. Rao), las149@pitt.edu (Laura A. Schaefer)

${ }^{1}$ Corresponding author, Tel.no. 412-624-9720 
discrete Boltzmann equation as a special finite-difference scheme [? ? ? ]. Consequently, the velocity-space can be discretized according to the flow-physics to be modeled. The discretization of space and time is a numerical requirement and, importantly, is not tied to the discretization of the velocity-space.

As a consequence, a subset of the LB method, called the off-lattice Boltzmann (OLB) method, was developed where space and time are independently discretized, i.e. $\Delta \boldsymbol{x} \neq \boldsymbol{\xi}_{i} \Delta t$. In the OLB method, we do not have the simplicity of a Lagrangian-type of evolution (streaming), rather the evolution of $f_{i}$ takes place in an Eulerian sense. The earliest OLB schemes were geared mainly towards extending the geometric flexibility of the LB method, which was previously limited, due to the requirement of a uniform Cartesian mesh. Several OLB schemes with different spatial discretization methods such as finite-volume (FV), finiteelement (FE), and finite-difference (FD), along with their variants, have been developed. For example, OLB schemes were used for non-uniform mesh [? ], curvilinear co-ordinates [? ? ], unstructured mesh [? ? ? ], finite element mesh [? ? ] among others. These advancements have made the LB method feasible for many practical engineering problems.

In addition to improving the geometric flexibility of the LB method, OLB schemes can also be used to solve the discrete Boltzmann equation (DBE) with higher-order lattices. Higher-order lattices are sets of discrete velocities, which are more suited to model more complex flows such as thermal flows, micro-scale (high Knudsen number) flows, etc. In many of these velocity sets (also termed as non-space-filling or offlattice), the discrete velocities cannot be expressed as an integer multiple of the smallest non-trivial speed. The D2Q16 velocity-set listed in [? ] and [? ] and the D2Q17 velocity-set in [? ] are typical examples. Since the regular stream-collide type of evolution scheme cannot be employed with these lattices, OLB schemes provide a viable evolution scheme for the DBE.

While several sophisticated spatial-discretization methods have been developed, many of the studies use time-marching schemes such as explicit Euler or Runge-Kutta (RK) for temporal discretization. Typically, these schemes require very small values of $\Delta t$ relative to the relaxation parameter $\tau$, to maintain numerical stability [? ? ]. This is in contrast to the LB method, which offers unconditional stability. Small $\Delta t$ requirement is particularly restrictive in the case of flows with high Reynolds number (Re) flows where $\tau$ is very small. Moreover, in the LB method, the Mach number $M a$ in the simulations has to be kept small (generally less than 0.1) to limit the compressibility errors. Small values of $M a$ lead to a slower convergence rate, especially for steady-state flow problems [? ? ]. Thus, the combined effects of small $M a$ and $\Delta t$ increase the overall computational cost of the RK-based OLB schemes.

Many alternative time-marching schemes have been proposed that maintain the numerical stability of the OLB method at higher values of $\Delta t$, relative to the relaxation parameter $\tau$, i.e. at higher $\Delta t / \tau$ values. These schemes vary greatly in their numerical stability due to the different approximations of the collision and advection part of the DBE. Hence, there is a need to systematically compare their relative performance in terms of the numerical stability of these schemes. This work addresses this need.

More specifically, we assess the stability of various OLB schemes, as quantified in terms of their maximum allowable $\Delta t / \tau$ ratio. This is done via benchmark testing on incompressible flow problems such as Taylorvortex flow, Poiseuille flow and lid-driven cavity flow. The on-lattice D2Q9 velocity set, which is used here for evaluation purposes, is described in Section 2.1. The various time-marching (OLB) schemes used in the comparative analysis are described in brief in Section 2.2 . 


\section{Numerical Formulation}

\subsection{Discrete Boltzmann Equation}

The basis for all OLB schemes is the Boltzmann equation with the Bhatnagar-Gross-Krook collision approximation [? ], which is given as:

$$
\frac{\partial f}{\partial t}+\boldsymbol{\xi} \cdot \nabla f=-\frac{1}{\tau}\left(f-f^{e q}\right)
$$

where $f \equiv f(\boldsymbol{x}, \boldsymbol{\xi}, t)$ is the single-particle distribution function, $\nabla f \equiv \frac{\partial}{\partial x_{\alpha}}$ is the spatial gradient of $f, \boldsymbol{\xi}$ is the microscale velocity, $\tau$ is the relaxation time of the collision process, and $f^{e q}=f^{e q}(\boldsymbol{x}, \boldsymbol{\xi}, t)$ is the local Maxwell-Boltzmann (equilibrium) distribution function. Equation 1 is continuous in velocity and configuration $(\boldsymbol{x}, t)$ space. To discretize the velocity space $\boldsymbol{\xi}$, the equation is non-dimentionalized using a chosen speed of sound, and the resulting $f^{e q}$ is expanded in a Taylor-series of fluid velocity $\boldsymbol{u}$ up to secondorder. The discrete velocities are then obtained from the requirement that the lower-order hydrodynamic moments with respect to the truncated $f^{e q}$ satisfy the conservation of mass, momentum, and energy [? ? ]. Following this procedure, we obtain the widely-used discrete velocity set of the D2Q9 lattice, for which the discrete Boltzmann-BGK equation can be written as:

$$
\frac{\partial f_{i}}{\partial t}+\boldsymbol{\xi}_{\boldsymbol{i}} \cdot \nabla f_{i}=-\frac{1}{\tau}\left(f_{i}-f_{i}^{e q}\right)
$$

where $f_{i} \equiv f_{i}\left(\boldsymbol{x}, \boldsymbol{\xi}_{i}, t\right), f_{i}^{e q} \equiv f_{i}^{e q}\left(\boldsymbol{x}, \boldsymbol{\xi}_{i}, t\right)$ and $i=0,1,2 \cdots, 8$. Here, while the Greek subscripts $\alpha \equiv\{x, y\}$ in 2D imply summation, the Latin subscripts (over velocity) do not imply summation. Equation 2 is termed as the discrete Boltzmann equation (DBE), and for a general class of discrete velocities, also referred to as the discrete velocity model (DVM). The D2Q9 velocity set is given by:

$$
\boldsymbol{\xi}_{i}= \begin{cases}(0,0) & \text { for } i=0 \\ \sqrt{3} c_{s} \cos \left(\theta_{i}, \sin \theta_{i}\right) & \text { for } i=1,2,3,4 \\ \sqrt{3} c_{s}\left(\sqrt{2}\left(\cos \theta_{i}, \sin \theta_{i}\right)\right) & \text { for } i=5,6,7,8\end{cases}
$$

where $\theta_{i}=(i-1) \pi / 2$ for $i=1-4, \theta_{i}=(2 i-9) \pi / 4$ for $i=5-8$, and $c_{s}=1 / \sqrt{3}$ is the speed of sound in the lattice. Figure 1 shows a representation of the D2Q9 lattice.

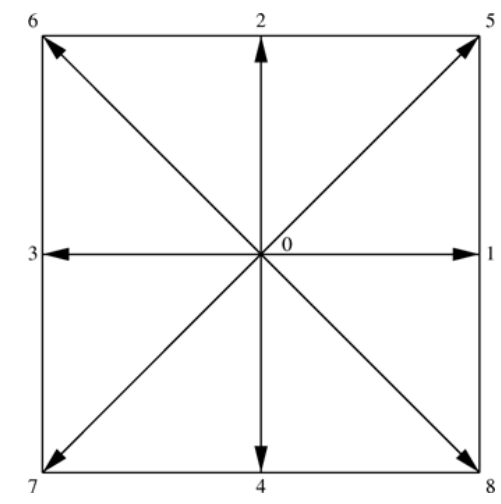

Figure 1: D2Q9 velocity lattice.

The discrete form of the equilibrium distribution function (EDF) is given by: 


$$
f_{i}^{e q}=\rho w_{i}\left[1+3 \frac{\boldsymbol{\xi}_{\boldsymbol{i}} \cdot \boldsymbol{u}}{c^{2}}+\frac{9\left(\boldsymbol{\xi}_{\boldsymbol{i}} \cdot \boldsymbol{u}\right)^{2}}{2 c^{4}}-\frac{3 \boldsymbol{u}^{2}}{2 c^{2}}\right]
$$

where the weights, $w_{i}$, are:

$$
w_{i}= \begin{cases}4 / 9 & \text { for } i=0 \\ 1 / 9 & \text { for } i=1,2,3,4 \\ 1 / 36 & \text { for } i=5,6,7,8 .\end{cases}
$$

The macroscale density and velocity are related to the DF through:

$$
\begin{aligned}
\rho & =\sum_{i=0}^{8} f_{i}=\sum_{i=0}^{8} f_{i}^{e q} \\
\rho \boldsymbol{u} & =\sum_{i=0}^{8} \boldsymbol{\xi}_{i} f_{i}=\sum_{i=0}^{8} \boldsymbol{\xi}_{i} f_{i}^{e q} .
\end{aligned}
$$

It can be shown that a Chapman-Enskog expansion with the above discrete form of the EDF recovers the incompressible, isothermal Navier-Stokes equation in the limit of small Knudsen and Mach numbers with a shear viscosity $\nu$ given by:

$$
\nu=c_{s}^{2} \tau
$$

where $\tau$ is the non-dimensional relaxation time.

\subsection{Off-Lattice Boltzmann Schemes}

\subsubsection{Explicit Runge-Kutta based schemes}

Since the discrete velocities $\boldsymbol{\xi}_{i}$ are constants, the DBE can be considered as a system of linear, first-order, ordinary differential equations (ODEs) with a weak source (collision) term. This assumption is generally valid only if the gradients of the conserved quantities in the flow are not too high, i.e., the collision term is not highly non-linear. The number of ODEs in the system equals the number of discrete velocities; for example; nine equations in case of the D2Q9 lattice. Therefore, in principle, commonly used time marching schemes for ODEs, such as Euler, Runge-Kutta, etc. can be employed for temporal discretization of Equation 1. On the other hand, FD or FV methods can be used for spatial discretization.

Focusing on temporal discretization, a general second-order Runge-Kutta (RK2) based OLB scheme for Equation 2 can be written as:

$$
\begin{aligned}
f_{i}^{n+\frac{1}{2}} & =f_{i}^{n}-\frac{\Delta t}{2} R_{i}^{n} \\
f_{i}^{n+1} & =f_{i}^{n}-\Delta t R_{i}^{n+\frac{1}{2}}
\end{aligned}
$$

where

$$
R_{i}^{n} \equiv-\left(\boldsymbol{\xi}_{i \alpha} \frac{\partial f_{i}^{n}}{\partial x_{\alpha}}\right)-\frac{1}{\tau}\left(f_{i}^{n}-f_{i}^{e q, n}\right)
$$

with the gradient term expanded in 2D Cartesian co-ordinates as: 


$$
\boldsymbol{\xi}_{i} \cdot \nabla f_{i}^{n}=\boldsymbol{\xi}_{i \alpha} \frac{\partial f_{i}^{n}}{\partial x_{\alpha}}=\xi_{i x} \frac{\partial f_{i}^{n}}{\partial x}+\xi_{i y} \frac{\partial f_{i}^{n}}{\partial y} .
$$

Here $f_{i}^{n+\frac{1}{2}} \equiv f_{i}\left(\boldsymbol{x}, t_{n}+\frac{\Delta t}{2}\right), f_{i}^{n+1} \equiv f_{i}\left(\boldsymbol{x}, t_{n}+\Delta t\right)$, etc. Similar expressions can be written for the fourth-order RK scheme (RK4) [? ]. In these schemes, the viscosity was related to the relaxation time through $\nu=c_{s}^{2} \tau$.

Many of the earliest OLB schemes employed the forward Euler, RK2 or RK4 schemes, in combination with a variety of spatial discretization schemes. Using the RK-based time marching schemes, the geometric flexibility of the LB method was extended to non-Cartesian domains, non-uniform grids, body-fitted, stretched grids, etc. [? ? ? ? ? ]. In the case of FD spatial discretization, the spatial order-of-accuracy can also be increased arbitrarily, using higher-order Taylor approximations of the gradient terms. On the other hand, several discrete velocity models with non-space-filling velocity-sets also employed the RK-based schemes as the evolution equation [?].

Despite the geometric flexibility made possible by the RK-based schemes, the size of the $\Delta t$ relative to $\tau$ has to be kept very small to maintain numerical stability. The constraint on $\Delta t$ comes primarily from the explicit approximation of the advection (LHS) and collision (RHS) terms of the DBE. An explicit advection approximation imposes a stability criterion on the size of $\Delta t$ through the $C F L$ condition, $C F L=\boldsymbol{\xi}_{i} \Delta t / \Delta x<$ 1. The $C F L$ condition is well-understood to be a necessary condition for the stability of advection type of equation. However, the overall stability of the scheme is governed by the more restrictive condition on $\Delta t$ due to explicit approximation of collision, given by the approximate condition $\Delta t<\tau$ [? ? ].

\subsubsection{Characteristics-based schemes}

The characteristics-based OLB schemes are based on time integration of the DBE along the characteristics using the $\theta$-method [? ? ]:

$$
\left.\tilde{f}_{i}^{n+1}=\tilde{f}_{i}^{n}+\Delta t\left[(1-\theta) \tilde{\Omega}_{i}^{n}\right)+\theta \tilde{\Omega}_{i}^{n+1}\right]
$$

where we denote $\Omega_{i}=\frac{1}{\tau}\left(f_{i}-f_{i}^{e q}\right)$ for brevity; a tilde indicates a term on the characteristic line, i.e., $\tilde{f}_{i}^{n+1}=f_{i}\left(\boldsymbol{x}+\boldsymbol{\xi}_{i} \Delta t, t+\Delta t\right), \tilde{f}_{i}^{n}=f_{i}(\boldsymbol{x}, t)$; and $0 \leq \theta \leq 1$. For $\theta=\left\{0, \frac{1}{2}, 1\right\}$, we obtain an explicit $\mathcal{O}(\Delta t)$, an implicit $\mathcal{O}\left(\Delta t^{2}\right)$, and an implicit $\mathcal{O}(\Delta t)$ approximation of the collision term, respectively. The $\mathcal{O}(\Delta t)$ schemes are Euler-type schemes, and the $\mathcal{O}\left(\Delta t^{2}\right)$ is a Crank-Nicholson-type scheme. However, a $\mathcal{O}\left(\Delta t^{2}\right)$ collision approximation can still be obtained for any value of $\theta \in[0,1]$, if the viscosity and relaxation time are related through:

$$
\nu=\left(\frac{\tau}{\Delta t}-0.5+\theta\right) c_{s}^{2} \Delta t
$$

A distinction should be made on the order of magnitude of $\tau$ as used in the standard LBM versus in the OLB method. In the standard LBM, $\tau$ is $\mathcal{O}(1)$, and in fact typically in the range of $0.6<\tau<3.5$. In the OLB method, on the other hand, due to non-dimentionalization, $\tau$ is $\mathcal{O}(K n)$, where $K n$ is the Knudsen number. Therefore, in OLB method, $\tau$ is $\mathcal{O}(0.01)$ or lower, depending upon the $R e$.

Broadly based on Equation 11, several OLB schemes have been proposed that are numerical stable at much larger $\Delta t / \tau$. In general, these schemes differ in their advection and collision approximations (explicit or implicit), as described below.

Following Equation 11, Lee and Lin [? ] proposed a fully-explicit scheme (advection and collision), termed herein as $\mathrm{AE} / \mathrm{CE}$, which can be written as: 


$$
\begin{aligned}
f_{i}^{n+1}= & f_{i}^{n}-\Delta t\left[\xi_{i \alpha} \frac{\partial f_{i}^{n}}{\partial x_{\alpha}}+\frac{1}{\lambda}\left(f_{i}^{n}-f_{i}^{e q, n}\right)\right]+\Delta t^{2} \xi_{i \alpha} \frac{\partial}{\partial x_{\alpha}}\left[\frac{1}{2} \xi_{i \beta} \frac{\partial f_{i}^{n}}{\partial x_{\beta}}+\frac{1}{\lambda}\left(f_{i}^{n}-f_{i}^{e q, n}\right)\right] \\
& -\frac{\Delta t^{3}}{2} \frac{1}{\lambda} \xi_{i \alpha} \frac{\partial}{\partial x_{\alpha}}\left[\xi_{i \beta} \frac{\partial}{\partial x_{\beta}}\left(f_{i}^{n}-f_{i}^{e q, n}\right)\right]
\end{aligned}
$$

with $\lambda=\nu / c_{s}^{2}+0.5 \Delta t$ corresponding to $\theta=0$, where $\lambda$ is also termed as the modified-relaxation parameter.

It is well-known in the ODE theory that an implicit approximation of the relaxation (collision) term is critical for numerical stability, especially for stiff equations (highly non-linear flows). Following this fact, a scheme similar to $\mathrm{AE} / \mathrm{CE}$ but with an implicit collision (CI) treatment i.e., $\theta=1 / 2$ was proposed in [? ], herein referred to as the AE/CI scheme. The AE/CI scheme, however, required an iterative predictorcorrector type of approximation for collision, which increases the computational cost and complexity of the simulations.

In order to avoid an iterative procedure to approximate an implicit-collision, a variable transformation is often employed in the LB method to mask the implicitness of the collision term [? ? ]. A similar technique is employed in the OLB context, where a new DF, $g_{i}$, is defined as:

$$
g_{i}(\boldsymbol{x}, t)=f_{i}(\boldsymbol{x}, t)-\Delta t \theta \Omega_{i}\left(f_{i}(\boldsymbol{x}, t)\right)
$$

Importantly, the variable transformation process preserves mass and momentum conservation, i.e., $\rho=$ $\sum_{i} f_{i}=\sum_{i} g_{i}$ and $\rho \boldsymbol{u}=\sum_{i} \boldsymbol{\xi}_{i} f_{i}=\sum_{i} \boldsymbol{\xi}_{i} g_{i}$. The variable transformation with $\theta=\frac{1}{2}$ also maintains $\mathcal{O}\left(\Delta t^{2}\right)$ accuracy of the collision approximation [? ].

With the variable transformation transformation technique and $\theta=\frac{1}{2}$, Guo and Zhao [? ] proposed an OLB scheme that can be written as:

$$
g_{i}^{n+1}=f_{i}^{n}-\Delta t \xi_{i \alpha} \frac{\partial f_{i}^{n}}{\partial x_{\alpha}}-\frac{\Delta t}{2 \tau}\left(f_{i}^{n}-f_{i}^{e q, n}\right) .
$$

In this scheme, although the collision is implicit and $\mathcal{O}\left(\Delta t^{2}\right)$, the advection is explicit and $\mathcal{O}(\Delta t)$ along the characteristics. Following Equation 12 for $\theta=1 / 2$, the viscosity-relaxation time relation in this scheme is $\nu=\tau c_{s}^{2}$. In this work, this scheme is referred as GZ scheme.

Bardow et al. [? ] combined the variable transformation technique, along with an explicit $\mathcal{O}\left(\Delta t^{2}\right)$ advection approximation along the characteristics to yield the BKG scheme:

$$
\begin{aligned}
g_{i}^{n+1} & =g_{i}^{n}-\Delta t\left[\xi_{i \alpha} \frac{\partial g_{i}^{n}}{\partial x_{\alpha}}+\frac{1}{\lambda}\left(g_{i}^{n}-g_{i}^{e q, n}\right)\right]+\frac{\Delta t^{2}}{2} \xi_{i \alpha} \frac{\partial}{\partial x_{\alpha}}\left[\xi_{i \beta} \frac{\partial g_{i}^{n}}{\partial x_{\beta}}+\frac{2}{\lambda}\left(g_{i}^{n}-g_{i}^{e q, n}\right)\right] \\
& -\frac{\Delta t^{3}}{2 \lambda} \xi_{i \alpha} \frac{\partial}{\partial x_{\alpha}}\left[\xi_{i \beta} \frac{\partial\left(g_{i}^{n}-g_{i}^{e q, n}\right)}{\partial x_{\beta}}\right] .
\end{aligned}
$$

Due to the implicit treatment of the collision term through variable transformation, the $\Delta t<\tau$ constraint no longer applies. The only constraint on $\Delta t$ is the $C F L$ condition due to explicit treatment of advection. The viscosity is related to the relaxation time through $\lambda=\nu / c_{s}^{2}+0.5 \Delta t$. A similar scheme called the unstructured lattice Boltzmann with memory (ULBEM) was proposed by [? ]. The various schemes described above are summarized in Table 1. 
Table 1: Summary of various off-lattice Boltzmann schemes. Here $E$ indicates explicit, and $I$ indicates implicit. Spatial order-of-accuracy for advection depends upon the spatial discretization method and schemes used, and hence is excluded.

\begin{tabular}{|c|c|c|c|}
\hline Scheme & Advection & Collision & $\nu-\lambda$ relation \\
\hline \hline RK2/RK4 & $\mathcal{O}\left(\Delta t^{2}\right) \mathrm{E}$ & $\mathcal{O}\left(\Delta t^{2}\right) \mathrm{E}$ & $\lambda=\nu / c_{s}^{2}$ \\
\hline $\mathrm{AE} / \mathrm{CE}$ & $\mathcal{O}\left(\Delta t^{2}\right) \mathrm{E}$ & $\mathcal{O}\left(\Delta t^{2}\right) \mathrm{E}$ & $\lambda=\nu / c_{s}^{2}+0.5 \Delta t$ \\
\hline $\mathrm{AE} / \mathrm{CI}$ & $\mathcal{O}\left(\Delta t^{2}\right) \mathrm{E}$ & $\mathcal{O}\left(\Delta t^{2}\right) \mathrm{I}$ & $\lambda=\nu / c_{s}^{2}$ \\
\hline $\mathrm{GZ}$ & $\mathcal{O}(\Delta t) \mathrm{E}$ & $\mathcal{O}\left(\Delta t^{2}\right) \mathrm{I}$ & $\lambda=\nu / c_{s}^{2}$ \\
\hline $\mathrm{BKG}$ & $\mathcal{O}\left(\Delta t^{2}\right) \mathrm{E}$ & $\mathcal{O}\left(\Delta t^{2}\right) \mathrm{I}$ & $\lambda=\nu / c_{s}^{2}+0.5 \Delta t$ \\
\hline
\end{tabular}

\subsection{Implementation aspects of a OLB scheme}

Equation 16 represents a typical temporal evolution scheme for the DFs used in the OLB method. Clearly, the usual stream-collide scheme type of evolution on the standard LBM is replaced by a procedure that involves solving a set of ODEs at each node. For the BKG scheme, the algorithm consists of the following steps:

1. Initialize $f_{i}^{0}$ and $g_{i}^{0}$ according to prescribed initial conditions, i.e., set $f_{i}^{0}=g_{i}^{0}=g_{i}^{e q, 0}$, where $g_{i}^{e q, 0}=$ $g_{i}^{e q}\left(\rho^{0}, \boldsymbol{u}^{0}\right) \cdot \rho^{0}$ and $\boldsymbol{u}^{0}$ are the given initial conditions.

2. For a particular time-step $n$, evaluate $g_{i}^{n}$ through Equation 14 (variable transformation).

3. Evaluate the gradient terms $\frac{\partial g_{i}^{n}}{\partial x}, \frac{\partial^{2} g_{i}^{n}}{\partial x^{2}}$, etc. In the case of FD spatial discretization, with a centraldifferencing scheme, these quantities can be computed as:

$$
\begin{aligned}
\frac{\partial g_{i}^{n}}{\partial x} & =\frac{g_{i}\left(x+\Delta x, y, t_{n}\right)-g_{i}\left(x-\Delta x, y, t_{n}\right)}{2 \Delta x}+\mathcal{O}\left(\Delta x^{2}\right) \\
\frac{\partial^{2} g_{i}^{n}}{\partial x^{2}} & =\frac{g_{i}\left(x+\Delta x, y, t_{n}\right)-2 g_{i}\left(x, y, t_{n}\right)+g_{i}\left(x-\Delta x, y, t_{n}\right)}{\Delta x^{2}}+\mathcal{O}\left(\Delta x^{2}\right)
\end{aligned}
$$

Similar expressions can be written for the first-order derivatives in the $y$-direction, and the second-order mixed derivatives $\frac{\partial^{2} g_{i}}{\partial x \partial y}[?]$. Other schemes such as upwind-differencing can also be employed.

4. Evaluate $g_{i}^{n+1}$ per Equation 16 .

5. Evaluate $\rho^{n+1}, \boldsymbol{u}^{n+1}$ and $g_{i}^{e q, n+1}$.

6. Evaluate $f_{i}^{n+1}$ per equation 14 .

7. If $\boldsymbol{u}^{n+1}$ has converged, then stop, if not, repeat steps 2-6.

\section{Numerical Tests}

To test the numerical stability of the various schemes, as a function of the maximum stable $\Delta t / \tau$, several steady and unsteady, incompressible, two-dimensional flows were simulated, and their results are presented in this section. Since the focus of this work is on evaluation of the temporal schemes, the simpler finitedifference method is used to discretize the spatial domains. For uniformity, Equation 17 is used to evaluate the derivatives in the gradient term for all the OLB schemes tested here. The central-difference scheme is chosen since they are less diffusive than other $\mathcal{O}\left(\Delta x^{2}\right)$ schemes. This minimizes the contribution of numerical diffusion to the overall stability of the scheme. The numerical stability of a scheme can be concluded by the maximum allowable $\Delta t$ for a particular $R e$ and grid size. In other words, for a particular flow problem, we fix the $R e$ (fixed $\tau$ ) and grid size (fixed $\Delta x$ ) and vary $\Delta t$, until the simulation becomes unstable.

The schemes were coded in $\mathrm{C}++$ using the Armadillo linear algebra library [? ], and parallelized for a shared-memory architecture using OpenMP. 


\subsection{Taylor Vortex Flow}

To test the stability and accuracy of various schemes without the artifacts of a boundary treatment, we first simulate the Taylor-Green vortex flow. This flow represents the unsteady flow of a freely decaying twodimensional vortex, and is often used to evaluate the effective viscosity and temporal and spatial accuracy of a scheme. Here, the flow is computed within a periodic square box defined as $-\pi \leq x, y \leq \pi$ with a uniform Cartesian mesh of size $100 \times 100$ on the domain. The analytical solutions of the flow-field are given by:

$$
\begin{aligned}
u(x, y, t) & =-u_{\text {ref }} \cos \left(k_{1} x\right) \sin \left(k_{2} y\right) \exp \left[-\nu\left(k_{1}^{2}+k_{2}^{2}\right) t\right], \\
v(x, y, t) & =u_{\text {ref }} \frac{k_{1}}{k_{2}} \sin \left(k_{1} x\right) \cos \left(k_{2} y\right) \exp \left[-\nu\left(k_{1}^{2}+k_{2}^{2}\right) t\right], \\
p(x, y, t) & =p_{\text {ref }}-\frac{u_{0}^{2}}{4}\left[\cos \left(2 k_{1} x\right)+\frac{k_{1}^{2}}{k_{2}^{2}} \cos \left(2 k_{2} y\right)\right] \exp \left[-2 \nu\left(k_{1}^{2}+k_{2}^{2}\right) t\right] .
\end{aligned}
$$

In our simulations, to minimize compressibility effects, the Mach number is set as 0.01 . The reference velocity is $u_{r e f}=M a \times c_{s}$, the reference density is $\rho_{r e f}=1$, the reference pressure is $p_{\text {ref }}=\rho_{\text {ref }} / c_{s}^{2}$, and the wave numbers $k_{1}$ and $k_{2}$ are constants set to 1.0 and 4.0, respectively. The non-equilibrium initialization scheme proposed by [? ] is used to initialize the DFs, and periodic boundary conditions are applied in the $x$ and $y$ directions. The Re of the flow is 100 .

Figures 2 and 3 show the horizontal and vertical velocity profiles at different times as obtained using the BKG scheme. The velocity profiles are shown for $t / t_{c}=0.5,1$, and 2 , where $t_{c}=\ln 2 / \nu\left(k_{1}^{2}+k_{2}^{2}\right)$ is the time at which the amplitude of the decay is halved. Importantly, these simulation have been obtained with $\Delta t=10 \tau$, where $\tau=\nu / c_{s}^{2}$. The corresponding $C F L$ number is 0.7 . This confirms the observations of high $\Delta t / \tau$ made in [? ]. Moreover, the average relative error as defined by:

$$
E=\frac{\sum_{y}\left(\left|u_{\text {num }}-u_{\text {exact }}\right|+\left|v_{\text {num }}-v_{\text {exact }}\right|\right)}{\sum_{, y}\left(\left|u_{\text {exact }}\right|+\left|v_{\text {exact }}\right|\right)}
$$

is $<1 \%$. Here $u_{\text {num }}$ is the simulated velocity, $u_{\text {exact }}$ is the analytical velocity, and the summation is over vertical plane at $x=0$. This result demonstrates that the BKG scheme successfully overcomes the $\Delta t<\tau$ restriction imposed by the RK-based OLB schemes. The only remaining restriction on $\Delta t$ is the local $C F L$ number which is due to an explicit advection approximation. 


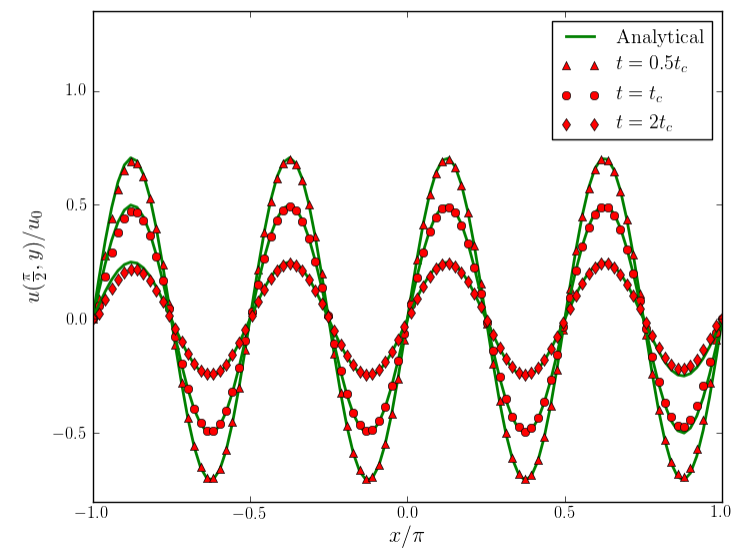

Figure 2: Horizontal velocity profile of Taylor-Vortex flow at various times. The numerical results have been obtained with a $\Delta t=10 \tau$ using the BKG scheme.

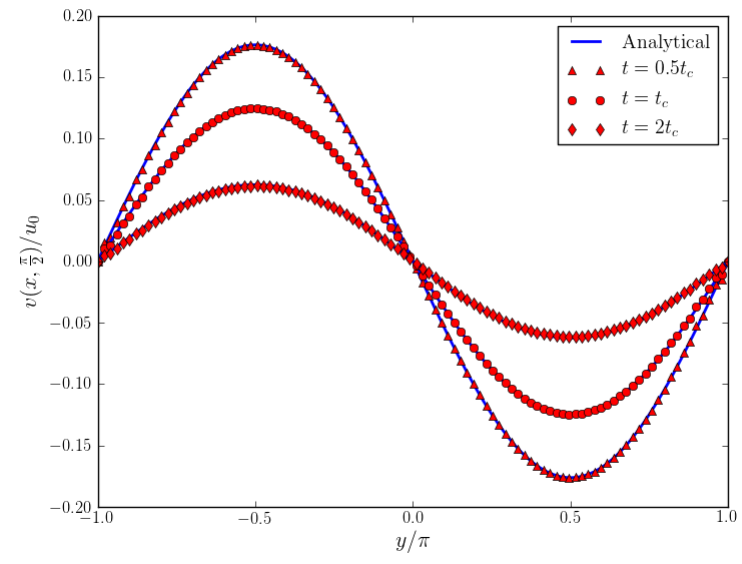

Figure 3: Vertical velocity profile of Taylor-Vortex flow at various times with $\Delta t=10 \tau$ using the BKG scheme.

For comparison, we also simulated the Taylor-Green vortex flow using the GZ and the AE/CE schemes. The GZ scheme, although having treated the collision term implicitly, could not achieve higher $\Delta t / \tau$ values. In fact, in their simulations, $\Delta t / \tau \approx 1.6$ for a $R e \approx 63$ with a mixed (central-upwind) differencing scheme. Furthermore, for a purely central-difference scheme, i.e., without the additional marginal stability of upwind schemes, the authors showed analytically using the von-Neumann stability analysis, that the maximum $\Delta t / \tau$ that can be attained is less than 1.5, and that at low $C F L$ numbers. We have observed that the $\mathrm{AE} / \mathrm{CE}$ permits $\Delta t>\tau$, but stable simulations are obtained at much lower values of $C F L$ number $(C F L<0.2)$ compared to the BKG AE/CI scheme.

\subsection{2-D Plane Poiseuille Flow}

Another useful numerical test is Poiseuille flow. A plane Poiseuille flow describes the steady, laminar flow of an incompressible fluid in a rectangular channel driven by a pressure gradient. Assuming symmetry and incompressibility, it can be shown that for Poiseuille flow, the Navier-Stokes momentum equation reduces to:

$$
\mu \frac{\partial^{2} u}{\partial y^{2}}=\frac{\partial p}{\partial x}
$$

which has a exact steady state solution for velocity given by:

$$
\begin{aligned}
u(y) & =4 u_{\max } \frac{y}{H}\left(1-\frac{y}{H}\right) \quad \text { for } 0 \leq y \leq H \\
v(x, y) & =0
\end{aligned}
$$

where, $H$ is the channel height and $u_{\max }$ is the center-line velocity where the magnitude of velocity is the maximum. The Reynolds number of the flow is defined by $R e=u_{\max } H / \nu$. In a LB simulation, imposing a pressure difference by specifying inlet and outlet densities increases the compressibility errors [? ]. Therefore, the effect of $\Delta p$ is imposed on the flow through an equivalent body-force $F$. This force has the same effect as having a pressure-gradient in the channel which produces the chosen $u_{\max }$. This body force can be evaluated from $F=8 u_{\max } \rho \nu / H^{2}$. 
For the reference case, the BKG scheme is used to obtain the results presented here. In the simulation, we set $H=1$ and $L=1$, where $L$ is the channel length. The domain is discretized into a uniform Cartesian mesh of size $100 \times 100$. The Mach number based on $u_{\max }$ is set to 0.1732 , which corresponds to a $u_{\max }$ of 0.1. The velocity is initialized to zero everywhere and the average density is set to one. Since a steady-state flow is simulated, the equilibrium initial condition is applied to the DFs. A non-equilibrium extrapolation boundary scheme is applied on the no-slip top and bottom walls [? ], and a periodic boundary condition is applied at the inlet and outlets. Various $R e$ flows are simulated by varying the viscosity but keeping the $M a$ constant. The simulations are run until a steady state criterion, defined as:

$$
C=\frac{\sum_{i, j}\left|u_{i, j}^{n}-u_{i, j}^{n-50}\right|}{\sum_{i, j}\left|u_{i, j}^{n}\right|}<10^{-6}
$$

is attained. Here $u_{i, j}^{n} \equiv u\left(x_{i}, y_{j}, n \Delta t\right)$, where $n$ is the time-step number, and the summation is over the entire flow field.

The simulated steady-state stream-wise velocity profile for $R e=100$, along with the analytical solution is shown in Figure 4. Clearly, the simulated and the analytical values are in excellent agreement with each other. The inset shows the velocity profile close to the wall, which is also in close agreement to analytical values. This serves as validation for the applicability and accuracy of the non-equilibrium boundary condition used in the simulation. It is worth noting that the velocity profile was generated on a $100 \times 100$ grid with a $\Delta t=5 \tau$. Even at such a high $\Delta t$, the average relative error as defined earlier is $\sim 2 \%$.

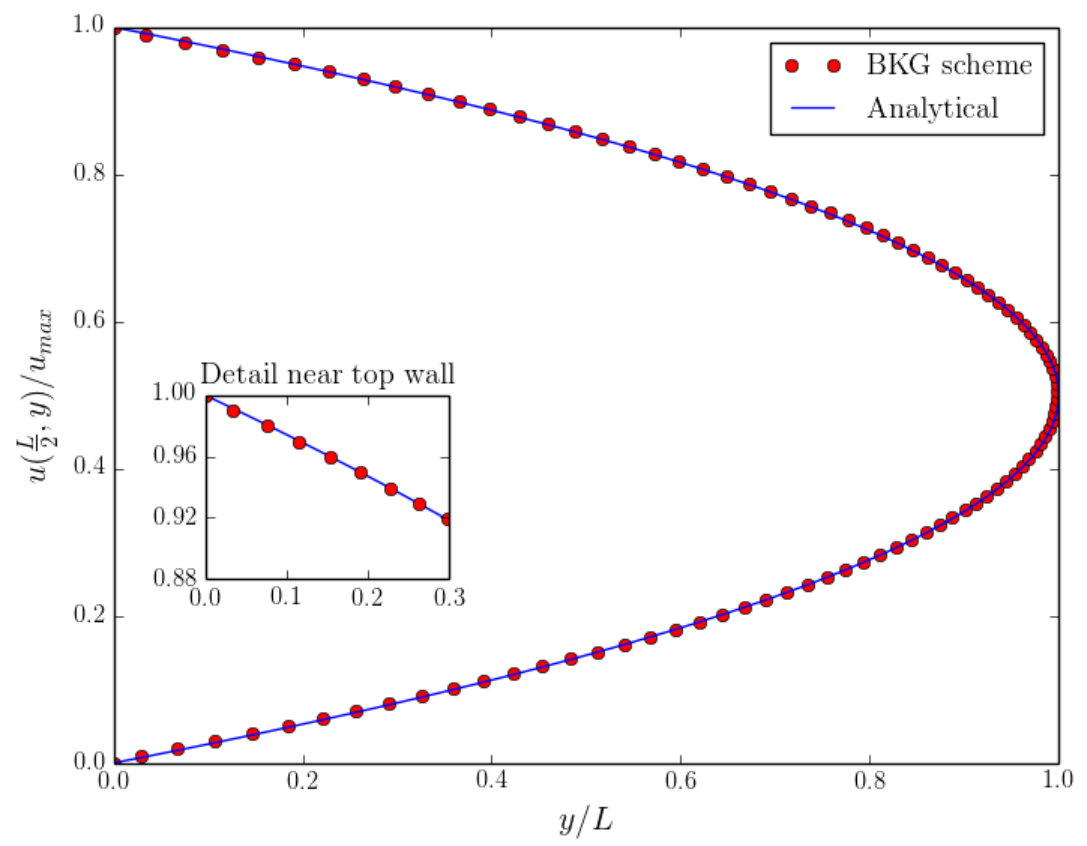

Figure 4: Steady-state horizontal velocity profile for Poiseuille flow at $R e=100$. The results were obtained using $\Delta t=5 \tau$ on a $100 \times 100$ mesh using the BKG scheme.

For comparison, the plane Poiseuille flow is also simulated using the AE/CE scheme, and the GZ scheme. 
Figure 5 shows the maximum allowable $C F L$ number versus $R e$ for the steady Poiseuille flow for the different schemes. This plot is obtained by fixing the grid size to $100 \times 100$ for each scheme, and then varying $\Delta t$. This is repeated for the various $R e$, as shown in the figure.

It can be seen that at low $R e$, both the $\mathrm{AE} / \mathrm{CE}$ and $\mathrm{BKG}$ schemes have almost similar maximum $C F L$ numbers. However, as Re increases, the maximum allowable $C F L$ number is clearly much higher with the BKG scheme, as compared to the AE/CE scheme. This enhanced stability at higher Re results from the implicit treatment of the collision term, which becomes highly non-linear as Re increases, thus directly benefiting from the local implicit treatment. Importantly, the implicit treatment of collision, allows for $\Delta t>\tau$, thus resulting in large $\Delta t$ and thereby reducing the computational effort.

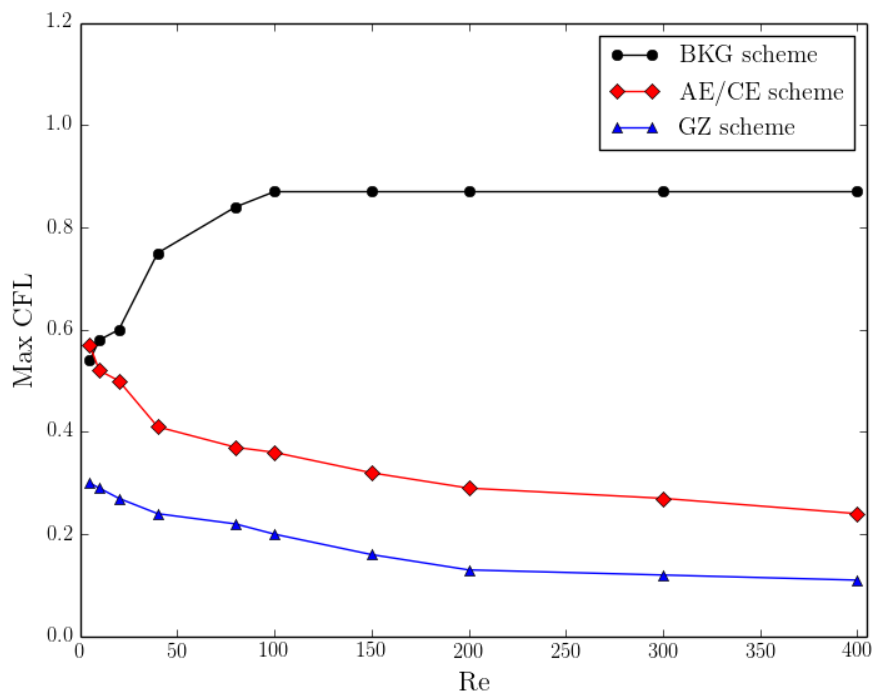

Figure 5: Maximum allowable $C F L$ number vs. Re for steady Poiseuille flow.

\subsection{Lid-Driven Cavity Flow}

Isothermal, 2-D lid-driven cavity flow is commonly used as a benchmark case to test and evaluate numerical schemes for incompressible viscous flows. The flow domain consists of a square cavity with three stationary walls on the sides and bottom, and a top wall (lid) that moves with a uniform tangential velocity $u_{\text {lid }}$. Despite the simple geometry, this flow exhibits many complex flow patterns such as formation of vortices near corners due to singularities. In our context, the lid-driven cavity case is also useful for quantitatively evaluating the effects of collision approximations (explicit/implicit) on flows with moderately large non-linearities (high $R e$ ), and thus quantifying the overall stability of different schemes.

The computational domain consists of a square with height $L=H=1$. The top wall moves with a constant velocity of $u_{l i d}=0.1$, and the Reynolds number of the flow is $R e=u_{l i d} H / \nu$. The domain is discretized on a uniform mesh of size $257 \times 257$. The non-equilibrium extrapolation scheme is applied for all of the walls. The flow is initialized by setting $\rho=1$ and $\boldsymbol{u}=0$ in the entire flow. The flow is simulated using four schemes: BKG, AE/CE, GZ, and also a RK2-based scheme.

As before, for the reference case, we present the results of simulations using the BKG scheme. Steady-state velocity components along the horizontal and vertical centerlines at various Re are shown in Figures 6 and 7 . 
These results have been obtained with $\Delta t=4 \tau$ and $C F L=0.5$. The results are compared with results from Ghia et al. who obtained their results with a $257 \times 257$ grid using the coupled strongly implicit multi-grid method, and a vorticity-stream-function formulation [? ] . The velocity profiles change from curved at lower $R e$ to linear for higher $R e$, which is consistent with the benchmark solutions. The near-linear velocity profiles at higher $R e$ in the central core of the cavity indicates a region of uniform vorticity.

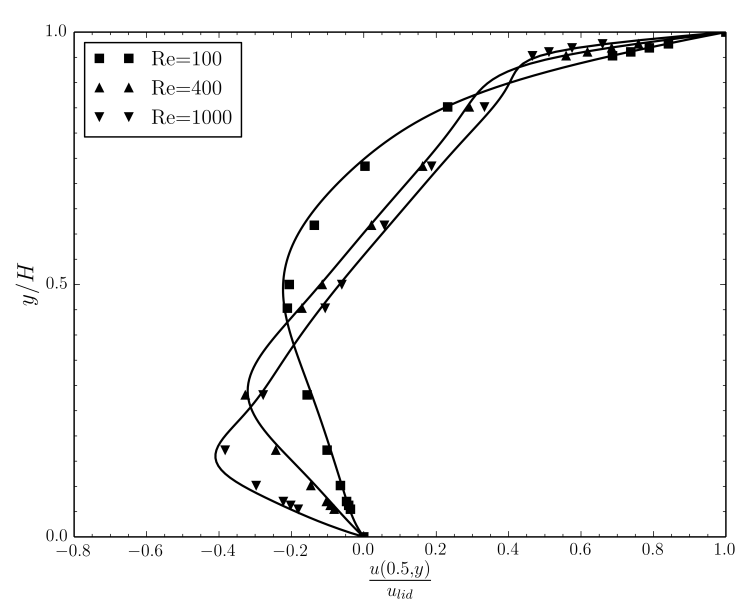

Figure 6: Centerline horizontal-velocity profile for a liddriven cavity at various $R e$. The solid lines indicate the simulated values, while the markers $(\boldsymbol{\square}, \mathbf{\Lambda}, \mathbf{\nabla})$ indicate the corresponding solution from Ghia et al. [?]

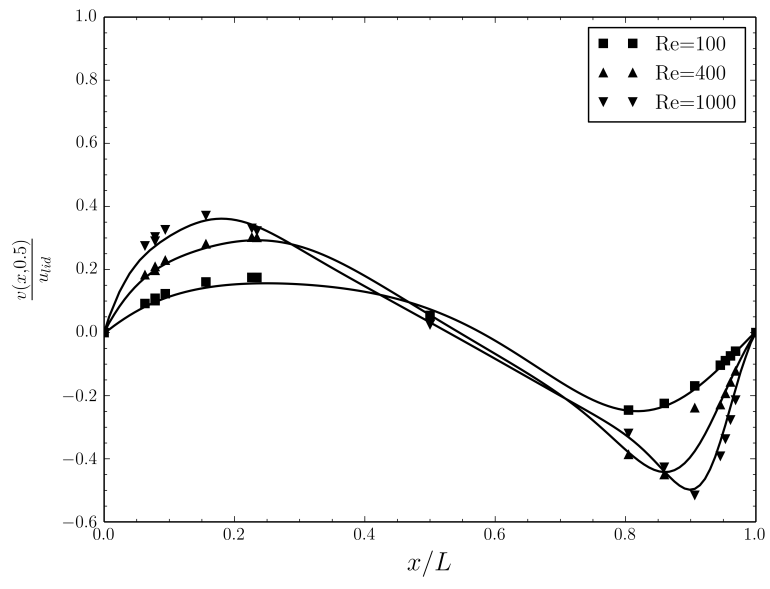

Figure 7: Centerline vertical-velocity profile for a liddriven cavity at various $R e$. The solid lines indicate the simulated values, while the markers $(\boldsymbol{\square}, \mathbf{\Lambda}, \boldsymbol{\nabla})$ indicate the corresponding solution from Ghia et al. [?]

To assess and quantify the effects of the size of $\Delta t$ on numerical stability, in Figures 8-13, we plot the stability region for each scheme, as determined by two parameters: $\Delta t / \tau$ and $\Delta t / \Delta x$ (please note that the scales of the axes vary across the figures). Broadly speaking, while $\Delta t / \tau$ represents the non-linear stability constraint imposed by collision, $\Delta t / \Delta x$ represent the linear-stability constraints of explicit advection. $\Delta t / \Delta x$ also represents the $C F L$ number in the case of a D2Q9 lattice. Hence, a map determined by these two parameters should serve a guide to gauge the overall numerical stability of the schemes with respect to size of $\Delta t$. In all of the maps, $\bullet$ indicates a stable solution and $\circ$ indicates an unstable solution. Figure 8 shows the stability region for the RK2-based OLB scheme with central differencing for $R e=100$. 


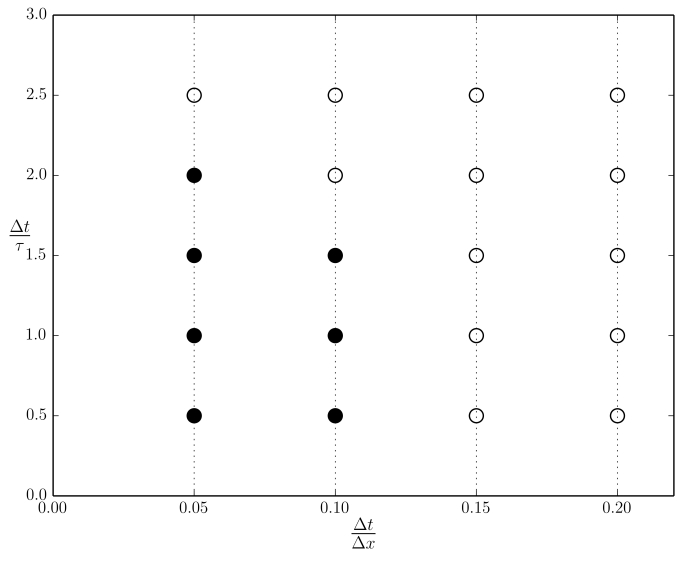

Figure 8: Stability region for the RK2 scheme at $R e=$ 100.

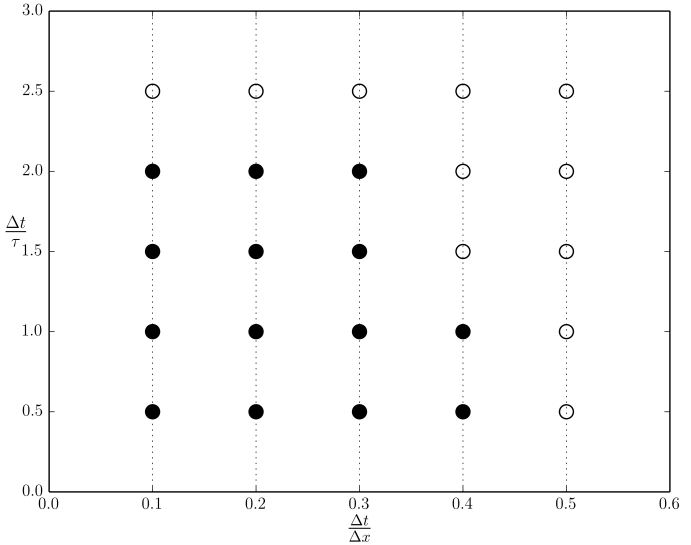

Figure 9: Stability region for the GZ scheme at $R e=100$.

From Figure 8, we can observe that the RK2-based scheme is unstable beyond $\Delta t / \tau>2$, irrespective of the $C F L$ number. Moreover, the stable solutions which are obtained at $\Delta t / \tau<2$ are done so at very low $C F L$ values. As described before, the small $\Delta t$ requirement stems from the fact that as $R e$ increases, the collision term becomes more non-linear and stiff, which requires an implicit approximation for numerical stability. Stability of RK2-based schemes can be increased slightly by adopting second-order upwind schemes; however, the marginal stability is added at the cost of increasing numerical diffusion. Similarly, multi-stage Runge-Kutta schemes such as RK4 can also increase stability. However, all RK-based OLB schemes involve multiple evaluation of the $f_{i}^{e q}$ term for each advancement in $\Delta t$, the number of evaluations depending upon the stages in the scheme. Since evaluation of $f^{e q}$ is a computationally intensive step, RK-based schemes are also computationally inefficient. Therefore, we can conclude that RK2 based OLB schemes are not particularly suitable for flows with large non-linearities.

Figure 9 shows the stability region for the GZ scheme for $R e=100$. From the stability-region plot, we can again see that the scheme is unstable for all $\Delta t / \tau>2$, irrespective of the $C F L$ number. Thus, in spite of the implicit collision approximation, large $\Delta t$ could not be used in the scheme. This observation is consistent with the stability analysis presented by the authors of the scheme.

The stability regions of the $\mathrm{AE} / \mathrm{CE}$ scheme are shown in Figure 10 and 11. From the figures, it is evident that the $\mathrm{AE} / \mathrm{CE}$ scheme does allow $\Delta t / \tau>2$, but does so only at lower $C F L$ number $(C F L<0.2)$. As $R e$ increases, however, the explicit collision approximation becomes inadequate, and hence the $\Delta t$ has to be smaller. 


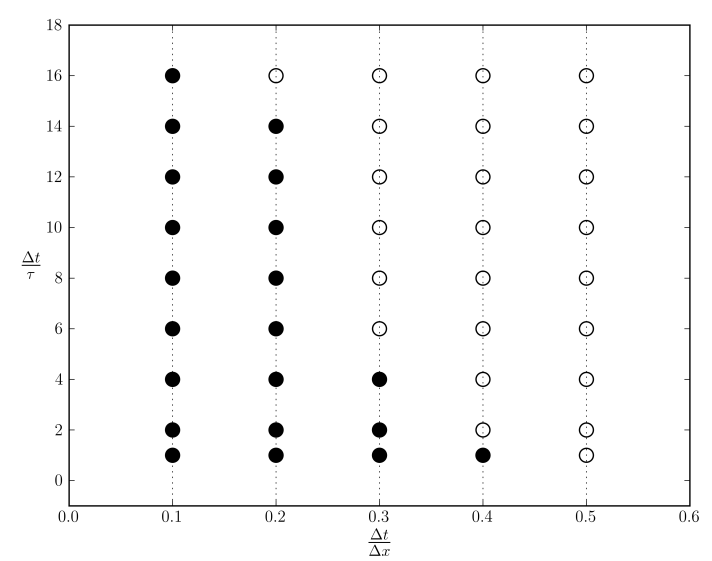

Figure 10: Stability region for the $\mathrm{AE} / \mathrm{CE}$ scheme at $R e=$ 400 .

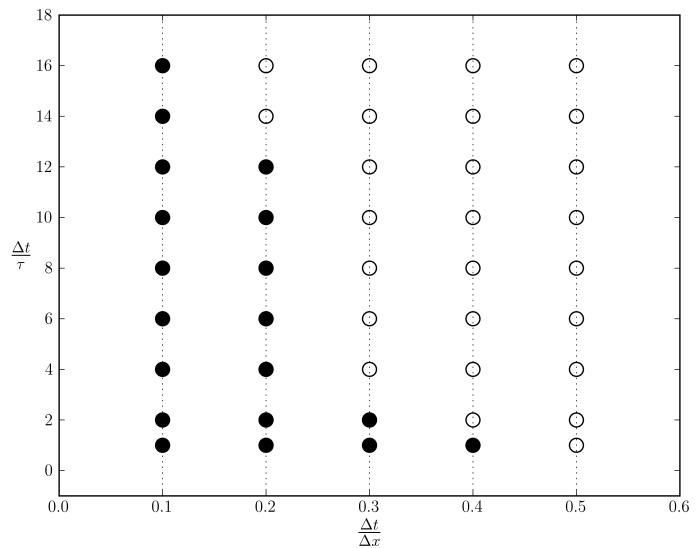

Figure 11: Stability region for the $\mathrm{AE} / \mathrm{CE}$ scheme at $R e=$ 1000 .

Finally, Figures 12 and 13 show the stability regions for the BKG scheme at $R e=400$ and 1000 . We can observe that the simulations are stable at $\Delta t / \tau$ as high as 30 , and that at high $C F L$ numbers. The trend also does not deteriorate with increasing $R e$ in the range that we have tested. This demonstrates the unconditional collision stability of the BKG scheme, with $\Delta t$ restricted only by the local $C F L$ number due to explicit advection.

The BKG scheme is also computationally more efficient than the comparable AE/CI scheme. This is because whereas a predictor-corrector type of scheme is needed for the implicit collision approximation in the $\mathrm{AE} / \mathrm{CI}$ scheme, a simple variable transformation given by Equation 14 is required in the BKG scheme. Additionally, the BKG scheme, as with all advection-explicit OLB schemes, retains the data-locality feature of the LB method, and hence can be easily parallelized.

Finally, it is important to note that we have discussed only the numerical stability of different OLB schemes in terms of maximum allowable $\Delta t$. However, as for all explicit advection schemes, including the BKG scheme, much smaller values of $\Delta \boldsymbol{x}$ and $\Delta t$ are required to get accurate solutions.

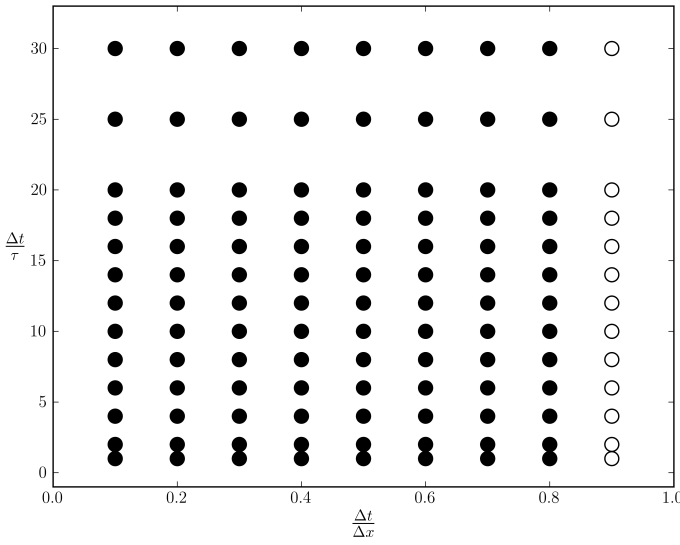

Figure 12: Stability region for the BKG scheme at $R e=$ 400.

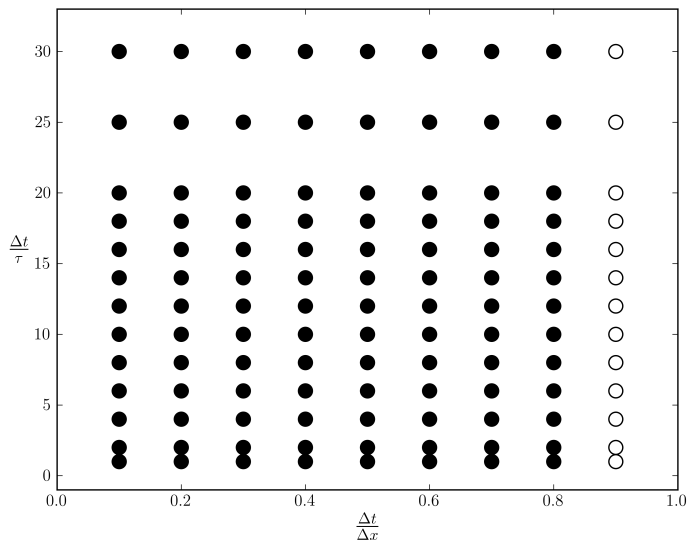

Figure 13: Stability region for the BKG scheme at $R e=$ 1000. 


\section{Conclusions}

In this work, several explicit OLB schemes have been compared by implementing them for benchmark flow problems. The following conclusions can be drawn from the observations:

1. The characteristics-based OLB schemes provide higher numerical stability compared to RK-based schemes.

2. In characteristics based-schemes, the $\Delta t<\tau$ constraint no longer applies, even at high Re.

3. The scheme proposed by Bardow et al. is stable over a much wider range of simulation parameters $(\Delta t / \tau, \Delta t / \Delta x)$ compared to other similar characteristics-based OLB schemes for the problems tested in this work. The BKG scheme also retains the simple explicit form of the LB method, while providing unconditional collision-stability.

These conclusions indicate that the BKG scheme provides the most stable and efficient explicit time-marching scheme, which can be extended to flow problems with FV or FD discretization. This scheme, in theory, can also be adopted for thermal problems with both off- and on-lattice discrete-velocity sets [? ].

\section{Acknowledgment}

This material is based upon work supported by the National Science Foundation under grant no. CBET1233106.

\section{References}

